\title{
Interventional Radiology Procedures after Pediatric Pyeloplasty and Ureteral Reimplantation in Patients with Postoperative Obstruction
}

\author{
Brent W. Snow ${ }^{1,2^{*}}$, M. Chad Wallis' ${ }^{1,2}$, G. Peter Feola ${ }^{2}$, John W. Rampton², \\ Teisha Shiozaki ${ }^{1}$ \\ ${ }^{1}$ Division of Pediatric Urology, University of Utah School of Medicine, Salt Lake City, USA \\ ${ }^{2}$ Primary Children's Medical Center, Salt Lake City, USA \\ Email: ${ }^{\text {brent.snow@hsc.utah.edu }}$
}

Received 13 May 2014; revised 10 June 2014; accepted 18 June 2014

Copyright (C) 2014 by authors and Scientific Research Publishing Inc.

This work is licensed under the Creative Commons Attribution International License (CC BY).

http://creativecommons.org/licenses/by/4.0/

(c) (i) Open Access

\section{Abstract}

Introduction: Obstructive complication after pyeloplasty or ureteral reimplant surgery is a rare though worrisome problem in pediatric urology. These are often complex patients with complicated post-operative courses that at times require interventional radiology procedures. The current literature is lacking in guiding principles to manage these complications. In this study we have reviewed these difficult to manage patients at our children's hospital over the past 15 years. Methods: A list of patients who underwent interventional radiology procedures to place nephrostomy tubes or internal double-J ureteral stents was compared a list of patients undergoing pyeloplasty or reimplant procedures. These lists were cross-referenced to a list of patients undergoing cystoscopic removal of double-J stents. This small patient group does not represent all complications but those with radiology intervention. Results: At our institution, during the years 1998-2011 we performed 458 pyeloplasties and 3003 open ureteral reimplant procedures. 14 (0.4\%) met all of the inclusion criteria. The long term outcome of these problems showed 11 of these patients went on to stability or improvement with either percutaneous drainage or JJ stent placement alone, and three of the reimplant patients ultimately required redo surgery. Of our pyeloplasty patients only three required percutaneous nephrostomy tube, and one went on to JJ stent placement $(0.66 \%$ of pyeloplasties). No patients in the pyeloplasty group needed surgical revision. Of patients how had undergone ureteral reimplantation, with or without tapering, seven of them underwent interventional radiology procedures $(0.23 \%$ of reimplant patients). Conclusion:

${ }^{*}$ Corresponding author.

How to cite this paper: Snow, B.W., Wallis, M.C., Feola, G.P., Rampton, J.W. and Shiozaki, T. (2014) Interventional Radiology Procedures after Pediatric Pyeloplasty and Ureteral Reimplantation in Patients with Postoperative Obstruction. Open Journal of Urology, 4, 87-90. http://dx.doi.org/10.4236/oju.2014.46015 
Pediatric urology patients with persistent obstruction after pyeloplasties and ureteral reimplantation surgery with or without tapering who needed interventional radiology rescue procedure resolved or stabilized in 11 of 14 patients. Surgical revision was performed in only 3 of our 14 patients after months of conservative trial after interventional radiologic procedures.

\title{
Keywords
}

\author{
Interventional Radiology, Pediatrics, Ureteral Reimplantation, Pyeloplasty, Postoperative \\ Obstruction
}

\section{Introduction}

Some of the most worrisome and vexing patients in pediatric urology are those who have obstructive complications after pyeloplasty or ureteral reimplantation surgery. These are often complex patients with complicated postoperative courses that sometimes acutely need interventional radiology. Other times the persistent obstruction is not recognized until later when interventional radiographic procedures are attempted [1]. One of the frustrations of clinicians who care for pediatric urology patients is not having a guideline or data in the literature to follow and rely upon to manage these difficult complications [2]-[8]. For this reason we have undertaken a review of this subset of obstructive complications following pyeloplasty or reimplant surgery at our children's hospital for the last 15 years that were managed with interventional radiology procedures.

\section{Materials}

After institutional review board approval, a list of patients who underwent interventional radiology procedures to place nephrostomy tubes or internal double-J ureteral stents was compared to a list of patients undergoing pyeloplasty or reimplant procedures. This list was also compared to a list of patients undergoing cystoscopic removal of double-J stents. It should be emphasized that this is not a comprehensive list of complications after these 2 procedures but this study was focused only on obstructive complications that underwent interventional radiology procedures. Symptomatic patients were evaluated promptly. Our clinical practice is to evaluate postoperative patients 6 - 12 weeks after surgery with an ultrasound which allowed us to detect clinically asymptomatic obstruction. The years of this study were 1998 to 2011 allowing for a minimum of 2 years of follow-up. Only patients who had undergone surgery with postoperative obstruction that had interventional radiology procedures were included in this study. Patients were exclude if follow-up occurred at other institutions or if medical circumstances were complicated by other comorbid problems such as renal transplantation or conjoined twins.

\section{Results}

During the years 1998 thru 2011 at our institution we performed 458 pyeloplasties and 3003 open ureteral reimplant procedures of all types. 14 (0.4\%) met all of our inclusion criteria. Table 1 shows the patient demographics. The male patients were slight younger than the female patients.

Table 2 indicates the initial type of procedure which was performed and led to unwanted complications and subsequent intervention. All but 2 of the procedures were performed with postoperative drainage. Indications for radiology intervention included obstructive symptoms (pain) and persistent or worsening hydronephrosis postoperatively. Seven of the 14 patients additionally had renal scans to evaluate their kidney function and drainage. Table 3 describes the timing of percutaneous nephrostomy tube placement. Some interventions were in the immediate postoperative period and others were delayed by several months. Five patients required additional intervention beyond conservative trial of nephrostomy tube drainage alone due to persistent or worsening hydronephrosis (Table 3).

The long term outcome of these complex and difficult problems showed 11 of these patients went on to stability or improvement with either percutaneous drainage or double $\mathrm{J}$ stent placement alone and three of the reimplant patients ultimately required redo surgery. Final outcome data is presented in Table 4. 
Table 1. Demographics.

\begin{tabular}{cccc}
\hline Gender & \# of Patients & Average Age (mo) & Age Range (mo) \\
\hline Males & 9 & 27 & $2.5-132$ \\
Females & 5 & 41.8 & $7-84$ \\
\hline
\end{tabular}

Table 2. Type of procedure.

\begin{tabular}{ccc}
\hline Procedure & Laterality & Drainage at Initial Procedure \\
\hline Pyeloplasty, $\mathrm{n}=3$ & $\mathrm{~L}=2, \mathrm{R}=1$ & 2 \\
Reimplant, $\mathrm{n}=7$ & $\mathrm{~B}=7$ & 6 \\
Tapered Reimplant, $\mathrm{n}=4$ & $\mathrm{~L}=2, \mathrm{R}=1, \mathrm{~B}=1$ & 4 \\
\hline
\end{tabular}

Table 3. Timing of percutaneous nephrostomy tube placement.

\begin{tabular}{cccc}
\hline Procedures & Average time to 1st IR Procedure & Range of time to 1st IR Procedure & 2 or more IR Procedures \\
\hline Pyeloplasty (3) & 12.6 days & 3 days -1 month & 1 \\
Reimplant (7) & 53 days & 2 days -11 months & 3 \\
Tapered Reimplant (4) & 28 days & 3 days -70 days & 1 \\
\hline
\end{tabular}

Table 4. Final outcomes.

\begin{tabular}{cc}
\hline Procedures & Outcomes \\
\hline Pyeloplasty & $1=$ stable mild, 1 = stable moderate, $1=$ no info \\
Reimplant & $4=$ improved, $1=$ improved on $\mathrm{R}$, worse on $\mathrm{L}, 1=$ stable moderate, $1=$ no info \\
Tapered Reimplant & $1=$ persistent severe, $3=$ stable moderate \\
\hline
\end{tabular}

\section{Discussion}

As the reader can see these are amongst the most difficult pediatric urology complications to deal with and there is little literature on clinical judgements from which to make good educated decisions. Fortunately these vexing complications are infrequent occurring in only $0.4 \%$ of our postoperative patients. Our practice in taking care of these patients has been to watch the patients clinically as long as they were stable and try all avenues of treatment before another surgical intervention is considered. This experience is a 15 year period (13 for patient accrual and 2 extra years for follow-up) for four pediatric urologists at our institution. Described in other terms, we averaged approximately one patient per pediatric urologist each fourth year so it is difficult to gain experience and insight for good clinical care.

It is uncommon for the medical literature to list series of difficult complications and how they were managed especially if the outcome is not totally favorable [2]. It was interesting to note that of our pyeloplasty patients only three required a percutaneous nephrostomy tube and one went on to double-J stent placement $(0.66 \%$ of pyeloplasties). Two patients improved with conservative drainage and one patient stayed unchanged but no patients in the pyeloplasty good were felt to need surgical revision.

Of the patients who have undergone ureteral reimplantation with or without tapering, seven underwent interventional radiology procedures $(0.23 \%$ of reimplant patients). If the patients did poorly and were symptomatic postoperatively in the hospital the procedures were done immediately. More commonly asymptomatic patients came back for a follow-up ultrasound at 6 - 12 weeks later as an outpatient and their persistent or worsening hydronephrosis was discovered at that time so an intervention was undertaken at that point. Of the seven patients who had nephrostomy tubes placed, three went on to placement of internal double-J stents for several months. Of the seven reimplant patients who had radiologic intervention three improved, one was unchanged and three worsened, going on to surgical revision. It should be noted that our conservative wait and see approach led some 
patients to second and third radiologic interventions. Even in those patients who had more than one intervention two of five patients improved or stabilized and did not undergo repeat surgery. It is our feeling from this data that at least one interventional radiology procedure including double-J stent placement and waiting several months if necessary is warranted. Clinical judgement must be used after that to determine whether further interventional procedures should be considered. After reviewing this data it is our opinion that surgical revision should be carefully considered after failure of the first conservative interventional radiologic procedure.

This study is difficult to interpret because none of these complicated patients were alike. Both pyeloplasty and reimplant procedures with or without tapering are remarkably successful in regards to postoperative obstruction. These complication rates must be viewed in their proper context since we sought only to look at patients who underwent interventional radiology procedures. Success for ureteral reimplantation in the context of this study can only be viewed as the absence of obstruction. Postoperative reflux was not assessed for this study. Patients who went directly on to redo pyeloplasty or reimplant would also not have been included.

The decision as to when and how to intervene is based on the individual patient circumstances. Complications, especially after reimplant procedures, can be bilateral so the timing for intervention can be affected by the magnitude of the obstruction or if both kidneys are involved. Some of these obstructions would be considered acute and would be expected to resolve rapidly while others were discovered later and expectations for rapid resolution may not be as high.

\section{Conclusion}

Postoperative obstruction after pyeloplasties and ureteral reimplantation surgery with or without tapering in pediatric urology patients who needed interventional radiology procedure resolved or stabilized in 11 of 14 patients. Postoperative obstruction at the ureteropelvic junction or the ureterovesical junction had a relative high likelihood of stabilization or improvement after radiologic intervention. Surgical revision was performed in only 3 of our 14 patients after months of conservative trial after interventional radiologic procedures.

\section{References}

[1] Uppot, R.N. (2009) Emergent Nephrostomy Tube Placement for Acute Urinary Obstruction. Techniques in Vascular and Interventional Radiology, 12, 154-161. http://dx.doi.org/10.1053/j.tvir.2009.08.010

[2] Rosman, B.M., Passerotti, C.C., Kohn, D., Recabal, P., Retik, A.B. and Nguyen, H.T. (2012) Hydronephrosis Following Ureteral Reimplantation: When Is It Concerning? Journal of Pediatric Urology, 8, 481-487. http://dx.doi.org/10.1016/j.jpurol.2011.10.017

[3] Charbonneau, S.G., Tackett, L.D., Gray, E.H., Caesar, R.E. and Caldamone, A.A. (2005) Is Long-Term Sonographic Followup Necessary after Uncomplicated Ureteral Reimplantation in Children? The Journal of Urology, 174, 14291431; Discussion 31; Author Reply 31-2. http://dx.doi.org/10.1097/01.ju.0000173128.73742.bc

[4] Falkensammer, M.L., Gobet, R., Stauffer, U.G. and Weber, D.M. (2008) To Cohen and Forget? Evaluation of Postoperative Imaging Studies after Transtrigonal Ureteric Reimplantation for Vesicoureteric Reflux in Children. Urologia Internationalis, 81, 218-221. http://dx.doi.org/10.1159/000144065

[5] Barrieras, D., Lapointe, S., Reddy, P.P., Williot, P., McLorie, G.A., Bigli, D., et al. (2000) Are Postoperative Studies Justified after Extravescial Ureteral Reimplantation? The Journal of Urology, 164, 1064-1066. http://dx.doi.org/10.1016/S0022-5347(05)67251-5

[6] Bomalaski, M.D., Ritchey, M.L. and Bloom, D.A. (1997) What Imaging Studies Are Necessary to Determine Outcome after Ureteroneocystostomy? The Journal of Urology, 158, 1226-1228. http://dx.doi.org/10.1016/S0022-5347(01)64436-7

[7] Braga, L.H., Lorenzo, A.J., Skeldon, S., Dave, S., Bagli, D.J., Khoury, A.E., et al. (2007) Failed Pyeloplasty in Children: Comparative Analysis of Retrograde Endopyelotomy versus Redo Pyeloplasty. The Journal of Urology, 178, 2571-2575; Discussion 5. http://dx.doi.org/10.1016/j.juro.2007.08.050

[8] Thomas, J.C., DeMarco, R.T., Donohoe, J.M., Adams, M.C., Pope IV, J.C. and Brock 3rd., J.W. (2005) Management of the Failed Pyeloplasty: A Contemporary Review. The Journal of Urology, 174, 2363-2366.

http://dx.doi.org/10.1097/01.ju.0000180420.11915.31 